\title{
Isothermal crystallization kinetics and morphology of nanodiamond/polyphenylene sulfide composites
}

\author{
Shuling Deng ${ }^{1, a}$, Chongmin Dü ${ }^{2, b}$, Hubin Lin ${ }^{2 c}$, Jianyi Zhu ${ }^{2, d}$, Zhidan Lin ${ }^{1, e}$ \\ ${ }^{1}$ College of Science and Engineering, Jinan University, China \\ ${ }^{2}$ Huizhou Changyi Science and Technology Co. Ltd, China
}

\begin{abstract}
Dsling123@163.com, 'bzchangyi@126.com, ${ }^{\mathrm{c}} 46674360 @ q q . c o m,{ }^{\mathrm{d}}$ chainz@sina.com, ${ }^{\mathrm{e}}$ linzd@jnu .edu.com
\end{abstract}

Keywords: Polyphenylene sulfide; Nanodiamond; Crystallization; Composites; Differential scanning calorimetry

\begin{abstract}
Nanodiamond (ND) as a filler material provides a nucleation effect on polyphenylene sulfide (PPS) and promotes its crystallization. The effects of ND on crystallization behavior and kinetics of composites were investigated by DSC. The results show that ND acts as an nucleating agent for PPS to accelerate the crystallization. And the existence of large amounts of ND in the PPS matrix may decrease isothermal crystallization activation energy.
\end{abstract}

\section{Introduction}

Polyphenylene sulfide (PPS) as a semi-crystalline high polymer, exhibites good thermal stability, chemical resistance, flame resistance, precision moldability, and excellent frictional properties. The outstanding characteristics of PPS make it used for numerous engineering applications, including electronics and electrical appliances, automobile, wear rings, and aerospace etc. [1,2]. However, the low glass transition temperature and its narrow processing temperature range, brittleness, and low strength restrict its further appplications. Therefore, PPS is mostly compounded with rigid particles [3-5] to obtain composites with a good comprehensive performance.

Nanodiamond (ND) possess porous surface, spherical particles, and other performances that provides broad application prospects in polishing, compound coatings, lubrication and medical science instruments [6,7]. So far, only a few reports have been published about the PPS composites filled with ND particles, especially about the crystallization of the composites of ND and polymers. In this manuscript, we prepared PPS/ND composites, examined the influence of ND on the isothermal crystallization behaviors and isothermal kinetics, and analyzed its variation, using a differential scanning calorimetry (DSC) technique.

\section{Materials}

PPS (HB relative density of $1.36 \mathrm{~g} / \mathrm{cm}^{3}$ ) was obtained from Deyang Science and Technology Company. ND (100nn) was supplied by Qinhuangdao Taiji Nano-products.

\section{Isothermal crystallization DSC curves of PPS/ND composites}

Figure 1 shows the isothermal crystallization curves of PPS and PPS composites containing $0.5 \mathrm{wt} \%$ and $2 \mathrm{wt} \%$ of ND crystallizing at various temperatures. As shown in Fig. 1, The peak shap and position of composites are different from that of pure PPS. As crystallization temperatures increase, the crystallization peaks become broader and shift to the right, suggesting that longer time required to complete crystallization.

At the same crystallization temperature, the intruduction of ND obviously reduced the tatal time of crystallization. When ND particle content in the composite was only $0.5 \mathrm{wt} \%$, the crystallization behavior of PPS composites changed greatly. It is mainly because ND with large specific surface area, 
provided more interaction interface, so that ND has strong adsorption to PPS molecular chain. These results indicate that ND particles provide a nucleating effect, acting as a nucleating agent. The effect shortens the crystallization induction period of DSC, improves crystallization rate, and reduces crystallization completion time.

\section{Isothermal crystallization kinetics of PPS/ND composites}

To further analyze the effect of ND on the crystallization behavior of PPS in the composites, Avramin equation[8-9] has been used to describe the isothermal crystallization process. The Avrami equation is as follows:

$$
X_{t}=1-\exp \left(-k t^{n}\right)
$$

Where $X_{t}$ is the relative crystallinity at crystallization time $t, k$ is Avrami crystallization rate parameter, and $n$ is the Avrami exponent depending on the nature of nucleation and growth geometry of the crystals. The double-logarithmic form of the Avrami equation is as follows:

$$
\ln \left[-\ln \left(1-X_{t}\right)\right]=\ln k+n \ln t
$$

$X_{t}$ is the relative crystallinity at crystallization time $t$, which can be calculated according to Eq.

$$
X_{t}=\int_{t_{0}}^{t}\left(d H_{C} / d t\right) \int_{t_{0}}^{t_{\infty}}\left(d H_{C} / d t\right) d t
$$

Where $t_{0}$ is the initial crystallization time; $t_{\infty}$ is infinite time; and $\mathrm{d} H_{c} / \mathrm{d} t$ is the heat evolution rate. From a plot of $\ln \left[-\ln \left(1-X_{\mathrm{t}}\right)\right]$ versus $\ln t$, a straight line is obtained. The exponent $n$ and crystallization kinetic constant $k$ can be obtained from the slope and intercept of the straight line, respectively. The half-time of crystallization $\left(t_{1 / 2}=(\ln 2 / k)^{1 / n}\right)$ is the temperature at which $X(\mathrm{t})$ is $50 \%$. The inverse of $t_{1 / 2}$, $\left(1 / t_{1 / 2}\right)$ can be used to characterize the crystallization rate

$$
G_{1 / 2}=1 / t_{1 / 2}
$$

According to Eq.(3), Fig. 2 illustrate the development of relative crystallinity with crystallization time for both neat PPS and its composites at different crystallization temperatures ranging from $234{ }^{\circ} \mathrm{C}$ to $242{ }^{\circ} \mathrm{C}$.

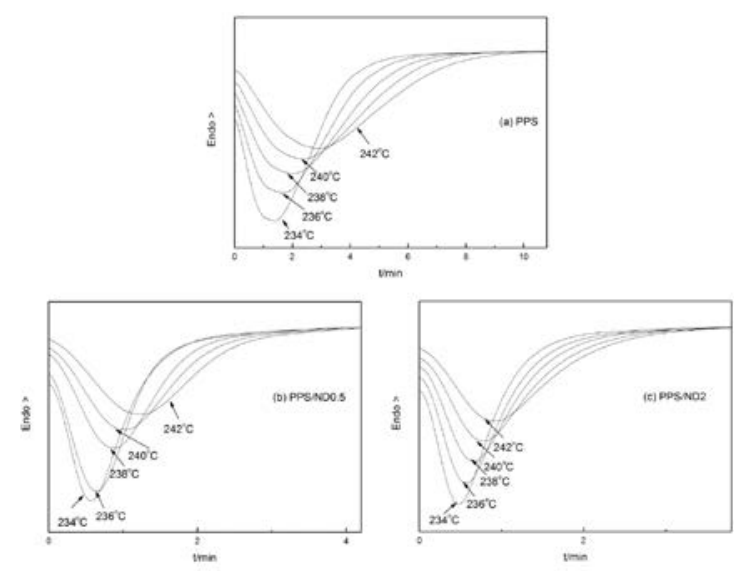

Fig.1

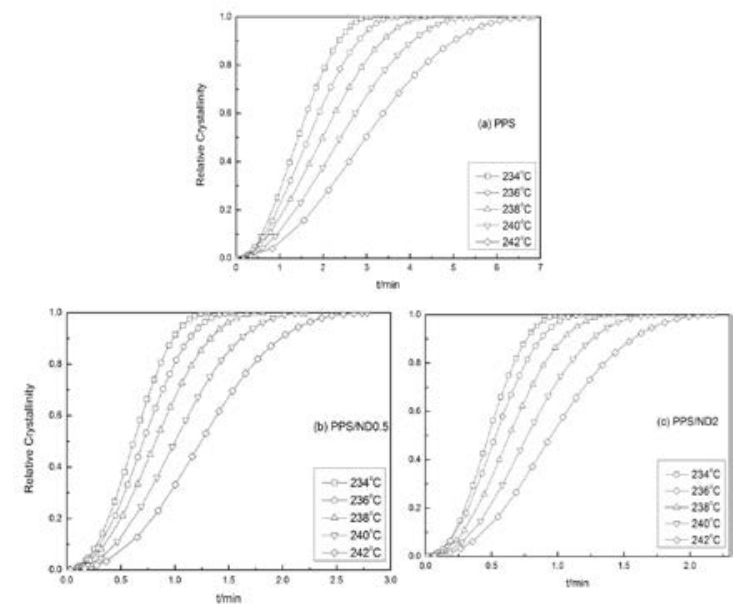

Fig.2

Fig.1 Isothermal crystallization curves of PPS and PPS/ND composites crystallizing at different temperature

Fig.2 Plots of relative crystallinity versus crystallization time at selected crystallization temperature for (a) neat PPS, (b) PPS/ND0.5, and (c) PPS/ND2

According diagrams of Fig. 2, plots of $\ln \left[-\ln \left(1-\mathrm{X}_{\mathrm{t}}\right)\right]$ versus $\ln$ for the neat PPS and its composites were drawn (Fig. 3). The obtained values of kinetic parameters of $k, n, t_{1 / 2}$ and $\mathrm{G}_{1 / 2}$ listed in Table1. The plots in Fig. 3 show linear relationship throughout the crystallization process, suggesting that the 
Avrami equation is suitable for describing the isothermal crystallization behavior of these composites. However, the later data points are not in perfect linear relationship, which indicates that the actual crystallization process of PPS composites is more complicated than Avrami model presented. This behavior is due to the fact that ND as a nanomaterial with large surface area, high surface energy, and easy reunion characters affects the crystallization rate of PPS.

Table 1 clearly shows that the $n$ values are varied between 1.01 and 1.09 for neat PPS and its two composites, indicating that the nucleation mechanism was not modified significantly in the PPS/ND composites at the selected $T_{c}$ range. It can be observed from the data that the crystallization rate constant $k$ of PPS decreased with increasing $\mathrm{T}_{\mathrm{c}}$, an indication of a strong dependence of $\mathrm{k}$ on the $\mathrm{T}_{\mathrm{c}} . k$ values of PPS/Nd composites also decreased with increasing crystallization temperature. Wheras $t_{1 / 2}$ of both near PPS and its composites increased at higher crystallizatiom temperature, indicating a slow-down of the crystal growth rate. At a same Tc, $t_{1 / 2}$ is greater in PPS than in PPS/ND0.5 and PPS/ND2composites , G is the opposite. It can be concluded that crystallization process of PPS is gradually accelerated in composites with increasing ND content due to the nucleation.
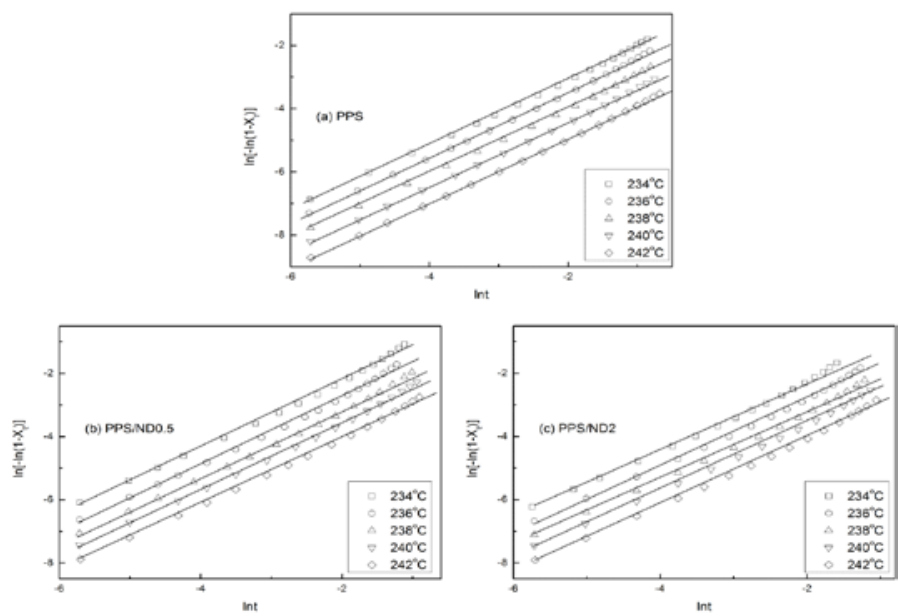

Fig.3 Avrami curves of PPS and PPS/ND composites

Table1 Kinetic parameters of PPS and PPS/ND composites

\begin{tabular}{ccccccc}
\hline Sample & $T_{\mathrm{c}}{ }^{\circ} \mathrm{C}$ & $n$ & $\mathrm{k} / \mathrm{min}^{-\mathrm{n}}$ & $t_{1 / 2} / \mathrm{min}$ & $G / \mathrm{min}^{-1}$ & $\triangle E / \mathrm{KJ} \mathrm{mol}^{-1}$ \\
\hline \multirow{5}{*}{ PPS } & 234 & 1.04 & 0.2274 & 1.42 & 0.704 & \\
& 236 & 1.02 & 0.1840 & 1.70 & 0.588 & \\
& 238 & 1.04 & 0.1560 & 2.08 & 0.481 & 233.56 \\
& 240 & 1.04 & 0.1204 & 2.46 & 0.407 & \\
& 242 & 1.01 & 0.0788 & 2.98 & 0.336 & \\
PPS/ND0.5 & 234 & 1.06 & 0.6281 & 0.66 & 1.515 & \\
& 236 & 1.06 & 0.4561 & 0.78 & 1.282 & \\
& 238 & 1.05 & 0.3260 & 0.90 & 1.111 & 256.31 \\
& 240 & 1.05 & 0.2531 & 1.05 & 0.952 & \\
& 242 & 1.03 & 0.1967 & 1.27 & 0.787 & \\
PPS/ND2 & 234 & 1.03 & 0.6415 & 0.51 & 1.961 & \\
& 236 & 1.09 & 0.5347 & 0.61 & 1.639 & \\
& 238 & 1.04 & 0.4029 & 0.70 & 1.429 & 218.87 \\
& 240 & 1.07 & 0.3570 & 0.83 & 1.205 & \\
& 242 & 1.06 & 0.2432 & 0.99 & 1.010 & \\
\hline
\end{tabular}

\section{The crystallization activation energy of PPS/ND composites}

According to Arrhenius model, crystallization activation energy can be expressed as Eq. (5)

$$
k^{1 / n}=k_{0} \exp \left[-\left(E_{2}-E_{1}\right) / R T_{c}\right]
$$


Where $E_{2}$ is crystalline state energy, $E_{1}$ is melt state energy, $k_{0}$ is pre-exponential factor, and $\mathrm{R}$ is the gas constant,. Substituting crystallization energy $\triangle$ for $E_{1}-E_{2}$ and taking logarithms on both sides of Eq.(5), we obtain Eq. (6)

$$
\ln k / n=\ln k_{0}+\Delta E / R T_{c}
$$

Plotting $\ln k / n$ against1/R $T_{\mathrm{c}}$ gives straight lines (Fig. 4). Calculating from the slopes of the straight lines, we obtain the values of $\Delta$ which listed in Table 1 . The obtained values of $\triangle E$ for neal PPS, PPS/ND0.5, and PPS/ND2 were 233.56, 256.31, and $218.87 \mathrm{KJ} \mathrm{mol}^{-1}$, respectively. $\triangle E$ of PPS is greater than this of PPS/ND2, but lower than this of PPS/ND0.5. The result reveals that the existence of large amounts of ND in the PPS matrix may reduce the mobility of PPS segments owing to the large surface area and special nanostructures of ND. It also suggests that, it is easier for polymer to crystallize, which proved that ND acts as a nucleating agent for PPS.

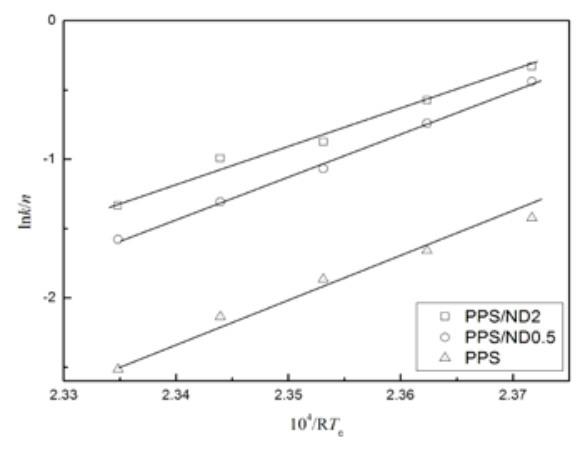

Fig.4 Relationships between $\ln k / n$ and $1 / \mathrm{R} T_{\mathrm{c}}$ of PPS and its composites

\section{Conclusion}

In summary, nanodiamond particles provided a heterogenous nucleation effecton PPS. DSC analysis suggested that ND accelerates the crystallization and shortens the crystallization time, the existence of large amounts of ND decreases isothermal crystallization activation energy of PPS.

\section{Acknowledgment}

This project was supported by Swan plan of Huizhou City, China (Grant No. 20131226120548921)

\section{References}

[1] A. Kalkar, V. Deshpande, M. Kulkarni, Polym Eng Sci 49 (2009) 397-417.

[2] M. Schuster, C.C. de Araujo, V. Atanasov, H.T. Andersen, K.D. Kreuer, J. Maier, Macromolecules 42 (2009) 3129-3137.

[3] A. Flores, M. Naffakh, A.M. Díez-Pascual, F. Ania, M.A. Gómez-Fatou, J Phys Chem C 117 (2013) 20936-20943.

[4] Y. Yang, H. Duan, S. Zhang, P. Niu, G. Zhang, S. Long, X. Wang, J. Yang, Compos Sci Technol 75 (2013) 28-34.

[5] A. Noll, T. Burkhart, Compos Sci Technol 71 (2011) 499-505.

[6] E. Osawa, Diam Relat Mater.16 (2007) 2018-2022.

[7] J.Y. Lee, D.S. Lim, Surf Coat Technol 188 (2004) 534-538.

[8] M. Avrami, J Chem Phys 8 (1940) 212-224.

[9] M. Avrami, J Chem Phys 9 (1941) 177-184. 